This item was submitted to Loughborough's Research Repository by the author.

Items in Figshare are protected by copyright, with all rights reserved, unless otherwise indicated.

Two-dimensional Ising model with competing interactions: phase diagram and low-temperature remanent disorder

PLEASE CITE THE PUBLISHED VERSION

http://dx.doi.org/10.1103/PhysRevB.79.014439

PUBLISHER

(C) The American Physical Society

VERSION

VoR (Version of Record)

LICENCE

CC BY-NC-ND 4.0

REPOSITORY RECORD

O'Hare, Anthony, F.V. Kusmartsev, and K.I. Kugel. 2019. "Two-dimensional Ising Model with Competing Interactions: Phase Diagram and Low-temperature Remanent Disorder”. figshare.

https://hdl.handle.net/2134/12784. 


\title{
Two-dimensional Ising model with competing interactions: Phase diagram and low-temperature remanent disorder
}

\author{
A. O’Hare, ${ }^{1}$ F. V. Kusmartsev, ${ }^{1}$ and K. I. Kugel ${ }^{1,2}$ \\ ${ }^{1}$ Department of Physics, Loughborough University, Leicestershire LE11 3TU, United Kingdom \\ ${ }^{2}$ Institute for Theoretical and Applied Electrodynamics, Russian Academy of Sciences, Izhorskaya Str. 13, Moscow 125412, Russia
}

(Received 14 September 2008; revised manuscript received 10 November 2008; published 30 January 2009)

\begin{abstract}
The two-dimensional Ising model with competing nearest-neighbor and diagonal interactions on the square lattice is studied by the transfer-matrix technique and by the Monte Carlo simulations. The phase diagram of this model is constructed with a special emphasis to the analysis of a glassy state arising as an order to disorder transition at low temperatures. Evidence of the glassy state (based, in particular, on the calculation of the average length of domain walls and on the Edwards-Anderson order parameter) and its characteristics are presented. It was shown that, in the frustrated Ising model, the domain-wall length correlates to the onset of the glassy state, that is, it may play the role of the order parameter for the Ising glass or for glasslike states in other frustrated magnetic systems.
\end{abstract}

DOI: 10.1103/PhysRevB.79.014439

PACS number(s): 75.10.Hk, 05.50.+q, 74.81.Fa, 03.67.Lx

\section{INTRODUCTION}

For several decades the low-dimensional magnetic structures, one dimensional (1D) and two dimensional (2D), have attracted widespread attention. The interest is partly stimulated by the studies of magnetic interactions in cuprate superconductors, where $\mathrm{Cu}-\mathrm{O}$ chains and planes in their undoped antiferromagnetic precursors can be considered as 1D and 2D Heisenberg antiferromagnets with spin one half. In a large number of such low-dimensional magnets, not only nearest-neighbor interactions but also next-nearest-neighbor ones play an important role. If the nearest-neighbor coupling constant $J$ is positive, in the absence of the next-nearestneighbor bonds $\left(J^{\prime}=0\right)$, the system is a simple antiferromagnet on bipartite lattices (e.g., a square lattice in 2D). The turning on next-nearest-neighbor coupling of the same sign $\left(J^{\prime}>0\right)$ leads to the frustration. The effects related to the interplay between frustration and quantum fluctuations in $2 \mathrm{D}$ quantum spin systems were actively studied in recent years both theoretically (see, e.g., Refs. 1-10 and references therein) and experimentally. ${ }^{11,12}$ These effects can lead to quantum phase transitions between magnetically ordered semiclassical and quantum paramagnetic ground-state phases such as glasses, whose nature and order parameter may be very nontrivial.

It is now well accepted that such a model with nearestneighbor $(J)$ and next-nearest neighbor $\left(J^{\prime}\right)$ for the spin-1/2 case on the 2D square lattice exhibits two phases with magnetic long-range order: one at small and another at large values of $J / J^{\prime}$. At small $J^{\prime}$, it has semiclassical Néel magnetic order with the magnetic wave vector $Q_{0}=(\pi ; \pi)$, whereas at large $J^{\prime}$, there appear antiferromagnetically coupled ferromagnetic chains (vertical or horizontal stripes) characterized by magnetic wave vectors $Q_{0}=(\pi ; 0)$ or $Q_{0}=(0 ; \pi)$. These two ordered phases are separated by an intermediate quantum paramagnetic phase without the longrange order, the nature of which is still under discussion.

It is interesting to note that the corresponding model with classical Ising-type spins at the 2D square lattice with competing nearest and next-nearest (diagonal) neighbor interac- tions exhibits similar features of the phase diagram. ${ }^{13}$ This similarity clearly demonstrates that frustrations play a fundamental role in low-dimensional magnetism, at least, not less important than quantum fluctuations.

A vivid example of such a system is given by planar clusters consisting of loops including a Josephson $\pi$ junction ( $\pi$-rings). A single $\pi$-ring is a superconducting loop consisting of Josephson junctions where at least one of them is a $\pi$ junction. ${ }^{14}$ Recently $\pi$-rings made of a combination of different high-temperature and low-temperature superconducting materials were deposited onto substrates in the form of one-dimensional and two-dimensional arrays. ${ }^{15,16}$ If there is one or an odd number of $\pi$ junctions in a loop, then the phase shift by $\pi$ results in doubly degenerate time-reversed ground states in the loop. Thus, a persistent supercurrent circulating in a clockwise or counterclockwise direction ${ }^{14}$ appears. Therefore, a phase shift by $\pi$ in such a junction results in the formation of an orbital current or a magnetic moment at the ring (see Ref. 14 for details). This means that a $\pi$-ring has the possibility of being used as a qubit and as an Ising spin variable.

A chain or a planar array of electrically isolated $\pi$-rings could be treated as a set of magnetic moments oriented perpendicular to the plane (Ising spins) and interacting via magnetic-dipole forces (in such geometry, these interactions will have an antiferromagnetic sign). Due to this dipole character of the interaction between the orbital moments, it is necessary to include the next-nearest-neighbor interactions or even other types of long-range interactions in addition to those between the nearest neighbors in the model. This dipole-dipole interaction may modify the values of the orbital magnetic moments and leads to a formation of the disordered and/or fractal structures in a one-dimensional chain. ${ }^{17,18}$

The ground state of a $\pi$-ring cluster depends on the coupling between the $\pi$-rings. Varying the couplings, one can obtain different ground states. For the conventional planar array of $\pi$-rings studied, for example, in Ref. 15 , the interaction between individual $\pi$-rings is mainly of the dipoledipole character and fixed. However, an introduction of ad- 


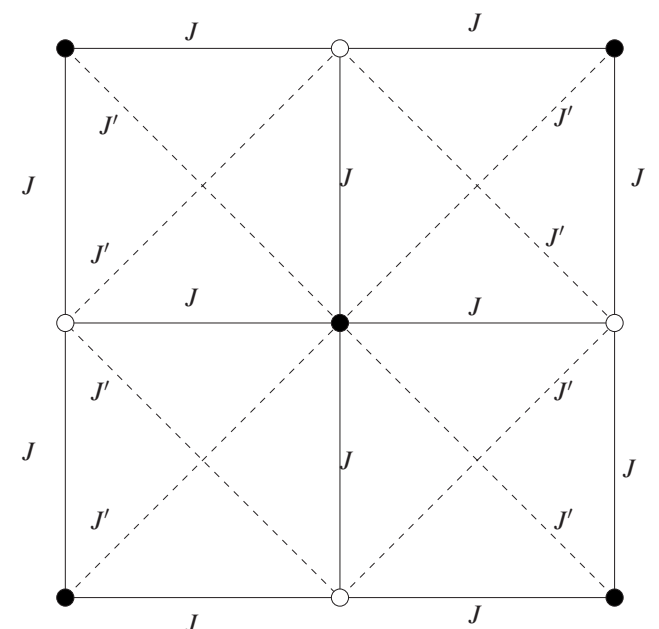

FIG. 1. Ising model with nearest-neighbor $J$ and diagonal $J^{\prime}$ interactions for the square lattice. Filled and open circles mean $s=+1$ and $s=-1$, respectively. Here, the usual two-sublattice arrangement of spins is shown.

ditional Josephson junction or a current loop located between the $\pi$-rings or other Josephson loops with persistent current may change this coupling significantly. For example, an introduction of an additional Josephson junction between two flux qubits, each consisting of three Josephson junctions, forms a well controllable coupling between these qubits. ${ }^{19}$

In our previous paper, ${ }^{13}$ we studied such a twodimensional Ising model with competing nearest-neighbor and diagonal interactions based on exact solutions for small square plaquettes and on the Monte Carlo simulations for large finite lattices. It was demonstrated that, in addition to the Néel, stripy, and intermediate states, there exists also an additional low-temperature glasslike phase characterized by the formation of large-scale inhomogeneities. It was demonstrated that the latter phase could be rather promising in the field of adiabatic quantum computing (AQC) where the states are switched adiabatically with the slow change in coupling constants.

In the present paper, we found that the glassy state of the Ising model discussed in Ref. 13 has an unambiguous description in terms of topological defects such as the domain walls. We argue that the length of the domain walls may play a role of the order parameter of this glassy state. We show that the temperature dependence of this length can provide an additional signature of the temperature behavior of the glassy state in frustrated lattices. The suggested order parameter defined as an average length of domain walls is significantly simpler and more transparent than the order parameter introduced by Edwards and Anderson. ${ }^{20}$ The suggested order parameter sheds also a light on the Monte Carlo simulations, which we have performed earlier. Moreover, in this paper we present a numerically exact solution of this frustrated Ising model using the transfer-matrix technique. We found that the formation of glassy state correlates with a degeneracy in the transfer-matrix spectrum. Such a technique allows us also to analyze the phase diagram of our system at large or even infinite square lattices based on accurate numerical solutions of the transfer-matrix equations.

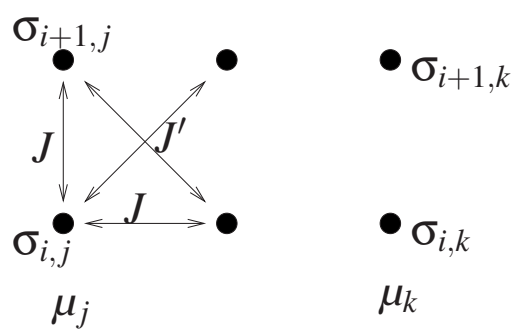

FIG. 2. Interactions between lattice columns in the 2D Ising model with next-nearest-neighbor interactions that define the transfer matrix.

\section{MODEL}

We start from the two-dimensional Ising model with antiferromagnetic nearest-neighbor and diagonal interactions. The Hamiltonian for such a model can be written as

$$
H=J \sum_{\langle i, j\rangle_{n n}} s_{i} s_{j}+J^{\prime} \sum_{\langle i, j\rangle_{d n}} s_{i} s_{j}-h \sum_{i} s_{i} .
$$

Here, we have $J, J^{\prime}>0, s$ is a two-value Ising variable $s= \pm 1,\langle i, j\rangle_{n n}$ and $\langle i, j\rangle_{d n}$ denote the summation over sites $i$ and $j$ being, respectively, nearest neighbors $(n n)$ and diagonal neighbors $(d n)$, and $h$ is the magnetic field in energy units. The geometry of the model is schematically illustrated in Fig. 1.

\section{TRANSFER MATRIX}

Let us begin by rewriting the Hamiltonian (1) for the $N \times N$ square lattice as

$$
H=\sum_{j=1}^{N}\left[E\left(\mu_{j}, \mu_{j+1}\right)+E\left(\mu_{j}\right)\right],
$$

where the summation is over all possible configurations involving site $j$, and the terms $E\left(\mu_{j}, \mu_{j+1}\right)$ and $E\left(\mu_{j}\right)$ are given by

$$
\begin{gathered}
E\left(\mu_{j}, \mu_{k}\right)=J \sum_{i=1}^{N} \sigma_{i, j} \sigma_{i, k}+J^{\prime} \sum_{i=1}^{N}\left(\sigma_{i, j} \sigma_{i+1, k}+\sigma_{i+1, j} \sigma_{i, k}\right), \\
E\left(\mu_{j}\right)=J \sum_{i=1}^{N} \sigma_{i, j} \sigma_{i+1, j}-h \sum_{i=1}^{N} \sigma_{i, j} .
\end{gathered}
$$

Here, $\mu_{j}$ denotes the set of spins in a column of the lattice $\mu_{j} \equiv\left\{\sigma_{1, j}, \sigma_{2, j} \cdots \sigma_{N, j}\right\}$. We can visualize the interactions between sites as in Fig. 2.

Writing out some terms of Hamiltonian (2) we can see that this notation does indeed include all the interactions in Hamiltonian (1): 


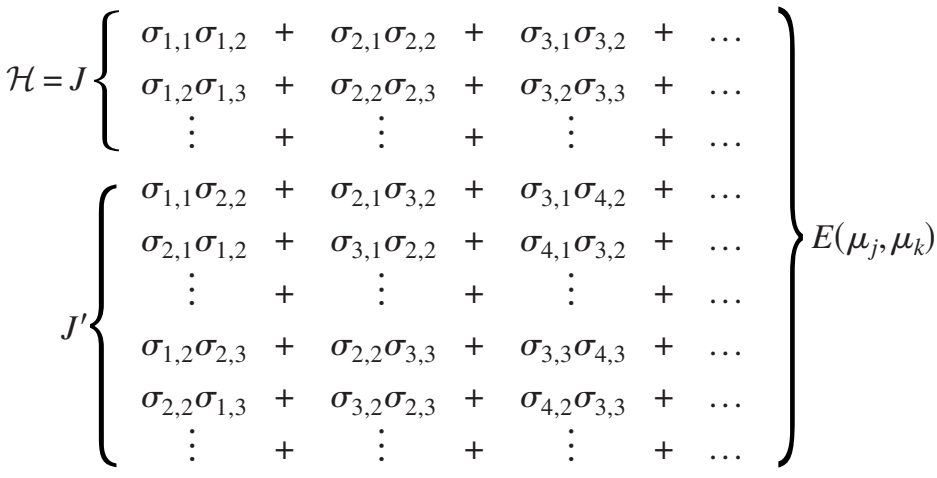

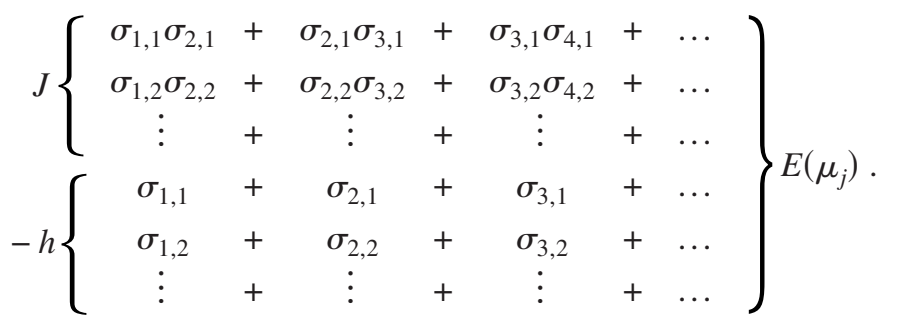

(2)

We can now define the following matrix for Hamiltonian

$$
\left\langle\mu_{j}|\mathbf{T}| \mu_{k}\right\rangle=\exp \left(-\beta\left[E\left(\mu_{j} \mu_{k}\right)+E\left(\mu_{j}\right)\right]\right) .
$$

Here, $\mathbf{T}$ is a $2^{N} \times 2^{N}$ transfer matrix, which is, in fact, a generalization of the well-known transfer matrix for the $2 \mathrm{D}$ Ising model with nearest-neighbor interactions. ${ }^{21}$ Using such transfer matrix, we can write the partition function $Z$ in a standard form

$$
\begin{aligned}
Z & =\sum_{\mu_{1}} \cdots \sum_{\mu_{N}}\left\langle\mu_{1}|\mathbf{T}| \mu_{2}\right\rangle\left\langle\mu_{2}|\mathbf{T}| \mu_{3}\right\rangle \ldots\left\langle\mu_{N}|\mathbf{T}| \mu_{1}\right\rangle \\
& =\sum_{\mu_{1}}\left\langle\mu_{1}\left|\mathbf{T}^{N}\right| \mu_{1}\right\rangle=\mathbf{T r} \mathbf{T}^{N} .
\end{aligned}
$$

In the thermodynamic limit $(N \rightarrow \infty)$, we have $\operatorname{Tr}\left(\mathbf{T}^{N}\right)=\lambda_{0}^{N}$, where $\lambda_{0}$ is the largest eigenvalue of matrix $\mathbf{T}$.

By the definition of transfer matrix $\mathbf{T}$, its matrix elements are non-negative. Therefore $\mathbf{T}$ belongs to a class of matrices that have a nondegenerate positive largest eigenvalue (the Perron-Frobenius theorem). ${ }^{22}$ It is not necessarily the case that the eigenvalues $\lambda_{i \neq 0}$ are real but the transfer-matrix method can be used nonetheless even if we employ the whole set of eigenvalues.

\section{PHASE DIAGRAM}

Our free parameters in the transfer matrix are the ratio of exchange constants, $J / J^{\prime}$, and the temperature, $T$, so we can create the transfer matrix at each value of $\left(J / J^{\prime}, T\right)$. Calculating the largest eigenvalue at each $\left(J / J^{\prime}, T\right)$ allows the partition function, $Z$, to be calculated as a function of the free parameters. From a knowledge of partition function, we can calculate the free energy written as

$$
\begin{aligned}
\mathcal{F} & =-k_{B} T \lim _{N \rightarrow \infty} \log Z \\
& =-k_{B} T \lim _{N \rightarrow \infty} \log \left[\lambda_{0}^{N}\left(1+\frac{\lambda_{1}^{N}}{\lambda_{0}^{N}}+\cdots\right)\right] \\
& \simeq-k_{B} T N \log \lambda_{0} .
\end{aligned}
$$

The entropy, $S$, and the specific heat at constant volume, $C_{v}$, can be calculated from the free energy according to the standard expressions $S=-T \frac{\partial \mathcal{F}}{\partial T}$ and $C_{v}=-T \frac{\partial^{2} \mathcal{F}}{\partial T^{2}}$.

It is not necessary, in general, to calculate the whole spectrum of eigenvalues for the transfer matrix as only the eigenvalue with largest modulus is needed to calculate the free energy, specific heat, and thus a phase diagram. Calculating the largest eigenvalue analytically for arbitrary size lattice is highly nontrivial, especially since the transfer matrix is not always symmetrical (and, as in the case of the Hamiltonian studied here, is usually nonsymmetric due to the nextnearest-neighbor interactions). Fortunately, several algorithms exist for calculating the largest eigenvalue of a matrix numerically.

Using the largest eigenvalue, we calculate free energy (6) and specific heat $C_{v}$ for the whole range of parameters $J / J^{\prime}$ and $T / J^{\prime}$. The results are presented in Fig. 3. One can see there the well-defined broad peaks, which have the lowest height in the parameter range, where the ratio $J / J^{\prime} \sim 2$. This is exactly the range where the frustration is the largest. Obviously, these peaks are associated with the conventional order-disorder phase transition where the disorder arises at higher temperatures. The peaks of the lowest height at the value $J / J^{\prime}=2$ might be an indication to a proliferation of topological defects and leading to disordered state at lower temperatures, which is in agreement with all our calculations.

Note that next eigenvalues of the transfer matrix $\left(\lambda_{1}, \lambda_{2}, \ldots\right)$ can be rather close in absolute value to $\lambda_{0}$, espe- 


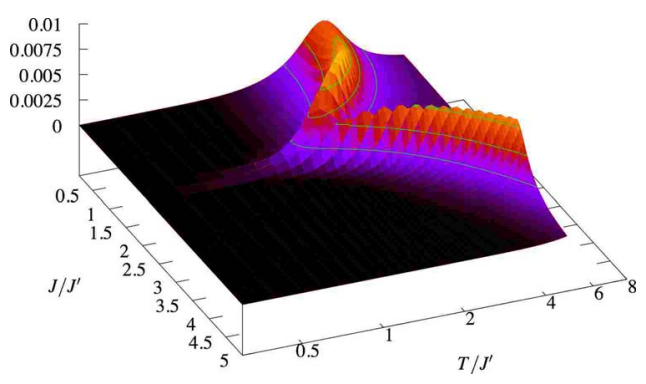

FIG. 3. (Color online) Specific heat for the for the Ising model with next-nearest-neighbor interactions on an $8 \times 8$ lattice calculated using the transfer-matrix method.

cially at low temperatures. However, the calculations with a full set of eigenvalues demonstrated that the plot presented in Fig. 3 remains nearly the same. This stems from the fact that, in the vicinity of the specific-heat peaks, $\lambda_{1}$ differs from $\lambda_{0}$ by a factor of about 0.5 at practically all values of $J / J^{\prime}$. This means that the contribution of net eigenvalues to the partition function of the $8 \times 8$ lattice does not exceed several percents.

We can draw a schematic phase diagram for the system from the knowledge of these peaks in the specific heat (see Fig. 4). We can see that there are three distinct regions in the phase diagram: a high-temperature phase and two lowtemperature phases separated by $J / J^{\prime}=2$. For $J / J^{\prime}<2$, we can see that stripe ordering is favorable while the Néel antiferromagnetic (checkerboard) ordering is favorable for $J / J^{\prime}$ $>2$. For $J / J^{\prime}=2$, we have a crossover from stripe to checkerboard antiferromagnetic ordering. This crossover could exist over a larger range of $J / J^{\prime}$ in practice where it would manifest itself by the formation of domains with either stripe or antiferromagnetic Néel-type ordering.

In a previous paper, ${ }^{13}$ we investigated this model using exact diagonalization for plaquettes of finite size where it

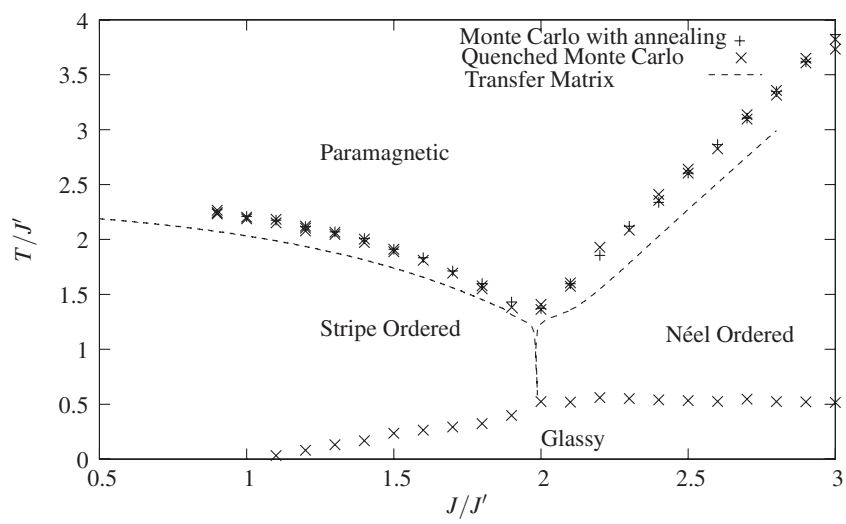

FIG. 4. Phase diagram for the Ising model with next-nearestneighbor interactions based on the transfer-matrix and Monte Carlo calculations. The peaks in the specific heat were used to determine the location of the phase transitions. The phase diagram for the transfer matrix and both types of Monte Carlo simulations (quenched and annealed) coincide at higher temperatures. However, an additional low-temperature glassy phase appears in the quenched Monte Carlo simulation; its boundary is also given by the order parameter related to the average length of the domain walls (see Sec. V). was found that the energies of defects and dislocations in the lattice were close to the ground state of this Hamiltonian. We concluded that it was possible for a carefully prepared system to become trapped in a low-temperature metastable state, and this was confirmed by Monte Carlo simulations of the model and by recent experiments on systems of $\pi$-rings, ${ }^{16}$ which may be modeled by the Hamiltonian presented here. In Fig. 4, we show the phase diagram of the Hamiltonian investigated in the present paper and compare it with the phase diagram from Monte Carlo simulations.

Two separate simulations were performed on a $16 \times 16$ lattice, averaging over ten runs using the Metropolis algorithm with 2500 thermalization steps and 25000 simulation steps in each run. In one simulation, we anneal the lattice to low temperature while in the second simulation, we quench the lattice by starting with a random initial state at each temperature. The phase diagram for the transfer matrix and both Monte Carlo simulations coincide at higher temperature. However, an additional phase appears in the quenched Monte Carlo simulation. We see that annealed and quenched Monte Carlo simulations describe well the thermally equilibrium states but the annealed Monte Carlo is not able to capture a glasslike state. Note also that both the transfer-matrix results and those given by the annealed Monte Carlo calculations correspond to the thermal equilibrium states so a good agreement between them is an indication to the accuracy of both approaches.

We performed simulations on lattices of various sizes over a subset of the $J / J^{\prime}$ values shown in Fig. 4 and did not found any qualitative difference in the location of the peaks in the specific heat. A $16 \times 16$ lattice turned out to be enough to capture the essential physics while maintaining sufficiently small errors in the calculations. In Ref. 13, we presented the calculations for the $100 \times 100$ lattice; they lead to qualitatively the same results although their accuracy is lower due to a smaller number of simulation steps used for the larger lattice.

\section{LOW-TEMPERATURE GLASSY STATE}

In Fig. 4, we see that the specific-heat data provided by the Monte Carlo simulations provide a compelling evidence of the low-temperature glassy state. However, the exact solution obtained by the transfer-matrix method in the previous section does not provide a direct evidence for such a glassy state. Although we found that, in the area of the phase diagram associated with the glassy state the largest eigenvalues become degenerate, it is still not clear how to express this finding in the form of the order parameter. Let us discuss this issue in more detail.

Here, we introduce a simple and transparent method allowing us to analyze the properties of a glasslike phase, which may reflect the appearance of the degeneracy in the transfer-matrix spectrum. The glassy state is treated as a set of small domains separated by domain boundaries. In such an approach, the length of domain boundaries can be considered as a measure of the "glassiness" of the system.

First of all, let us introduce a nonstandard definition of a domain boundary as a topological defect, which could help 

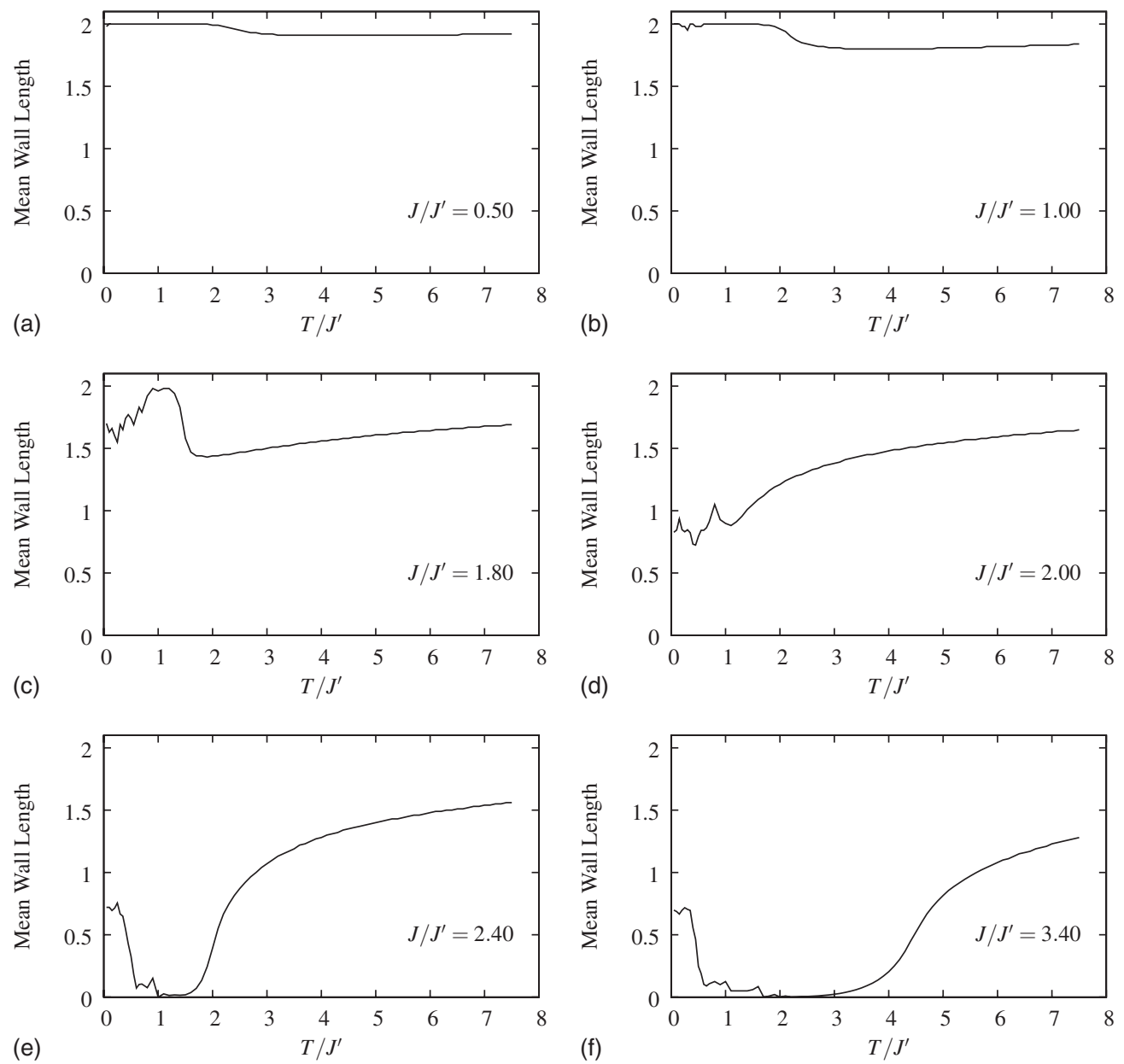

FIG. 5. Mean length of domain boundaries as function of temperature in the $2 \mathrm{D}$ frustrated Ising model at different values of parameter $\alpha=J / J^{\prime}$. The steep deviation of the mean length per lattice site from zero (at $\alpha>2$ ) and from two (at $\alpha<2$ ) is a signature of the onset of the glasslike state. Here, we present the results of Monte Carlo simulations without the annealing procedure (quenched system).

us in the further numerical analysis of the glassy state. Of course, in arguing that the domain boundary is a topological defect, we should first define the ordered state. It is natural to start with the usual two-sublattice ordering of Ising spins, where each site of one sublattice is surrounded by the sites of the other sublattice with the spins of opposite direction. Then, we can define a domain boundary as the boundary between two pieces of the ordered phase displaced with respect to each other. Hence, the uniform antiferromagnetic two-sublattice state has no domain boundaries. At the same time, the antiferromagnetic state with alternating ferromagnetic chains of opposite spins (horizontal or vertical stripes) may be treated as a state with maximally dense (with the period of one lattice constant) parallel domain boundaries.

Under such a definition of a domain boundary, we can also characterize an arbitrary disordered Ising state. For this purpose, let us introduce a measure of the disorder associated with the domain boundary length $l_{d}$. The simplest way to do this is to normalize such length per one lattice site. Then the antiferromagnetic state with horizontal or vertical stripes should be treated as a state with the domain boundary length $l_{d}=2$, whereas a classical two-sublattice antiferromagnetic Ising state will correspond to $l_{d}=0$. Then, for an arbitrary disordered state, the mean normalized length should fall within the $0<l_{d}<2$ range. Probably, the maximum disorder will correspond to $l_{d}=1$. Note that, at an applied magnetic field, the above inequality is violated. For example, in the saturated ferromagnetic state, we have $l_{d}=4$.

In Figs. 5 and 6, we show the temperature dependence of the domain boundary length at different values of the $J / J^{\prime}$ ratio. The results depend on the method employed in the simulation; in the first (quenched) simulation we start with a random initial state and run the thermalizing steps before taking measurements. We repeat the same steps for each temperature in the plot. In the annealing simulation we choose a random initial state for a high temperature, run the thermalizing steps, and take measurements. We then lower the temperature, keeping the state of the previous temperature at the end of the measurement steps as the initial state of the lower temperature. In this way, at low temperatures we always start our simulation in an ordered state, in which the system remains. In the quenched simulation, we see defects get "frozen" into the system at very low temperatures creating a cluster-glass state. In Fig. 5, we see that the quenched simulations exhibit a steep change in the mean length of domain walls at low temperatures. The corresponding onset temperature agrees well with that given by the low-temperature curve in Fig. 4. 

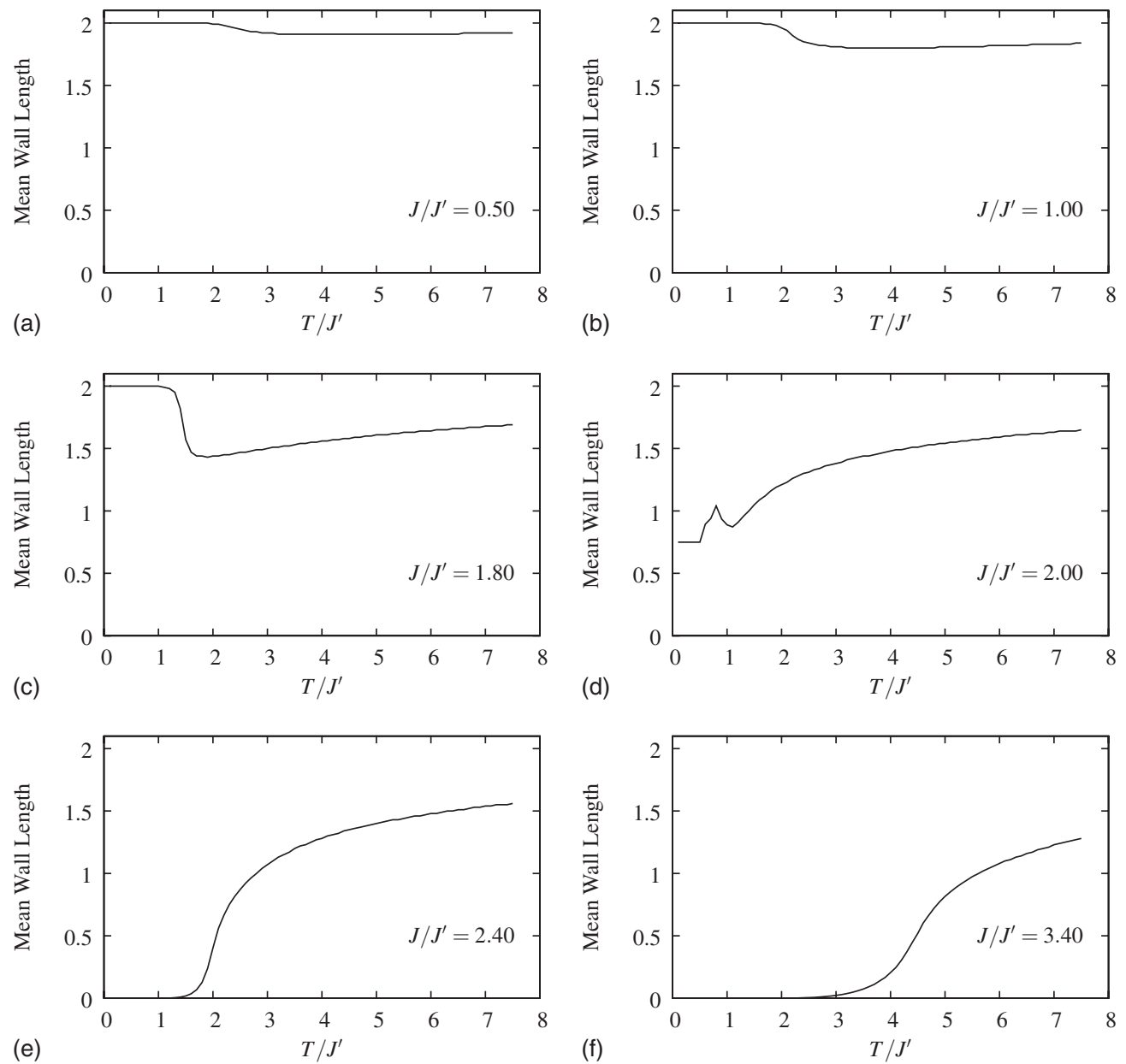

FIG. 6. Mean length of domain boundaries as function of temperature in the 2D frustrated Ising model at different values of parameter $\alpha=J / J^{\prime}$. Here, we present the results of Monte Carlo simulations with the annealing procedure (annealed system). Note that the annealing removes any signatures of low-temperature disorder except for $\alpha<2$, where the crossover between different types of the ground state occurs.

The glassy state can be also analyzed in a more traditional way. Indeed, in a typical antiferromagnet at high temperature, the system exists in a paramagnetic state where the global magnetic moment $m=\left\langle s_{i}\right\rangle$ is nonzero, where there is a slight imbalance between the number of "up" and "down" spins, and each spin is able to flip its orientation randomly. As the temperature is lowered the spins "freeze," in particular orientations, so that the global magnetization becomes zero as antiferromagnetic ordering develops. At low temperatures, the system should exist in an ordered state with long-range order. However, randomness reduces correlations to a few spins but temporal correlations due to freezing of the spins can be very strong. Local squared magnetization is given by the average of the autocorrelation functions. This is the order parameter proposed by Edwards and Anderson, ${ }^{20}$

$$
q_{\mathrm{EA}}=\lim _{t \rightarrow \infty} \lim _{V \rightarrow \infty}\left[\left\langle s_{i}\left(t_{0}\right) s_{i}\left(t_{0}+t\right)\right\rangle\right] .
$$

Spin-glass ordering, if it can be described within the framework of equilibrium statistical mechanics, corresponds to a nonzero value of the Edwards-Anderson order parameter. We can see from Fig. 7 that Monte Carlo simulations clearly show the Edwards-Anderson order parameter explod- ing as $T / J^{\prime} \rightarrow 0$. This is indicative of spin-glass ordering.

At the same time, when we use annealing to reach low temperatures, we have a situation where, at low temperatures, the system is in an ordered state (striped ordering at $J / J^{\prime}<2$ and the Néel-type antiferromagnetic ordering at $J / J^{\prime}>2$ ). If we were to anneal this system further, we would have a situation where the system is in an ordered ground state and where there is no energy available to create any frozen superstructures. This situation is illustrated in the right panel of Fig. 7 .

Thus, different types of the Monte Carlo simulations reveal the existence of the low-temperature glasslike phase with quite unusual properties. The glassy state is characterized by a proliferation of topological defects such as domain walls down to very low temperatures. That is why the appearance of the glassy state is well correlated with the change in the average length of domain walls, which play here a role of the order parameter for this glassy state.

\section{CONCLUSION}

In summary, we described a formation of disordered state arising at very low temperatures. The disorder stems from a 

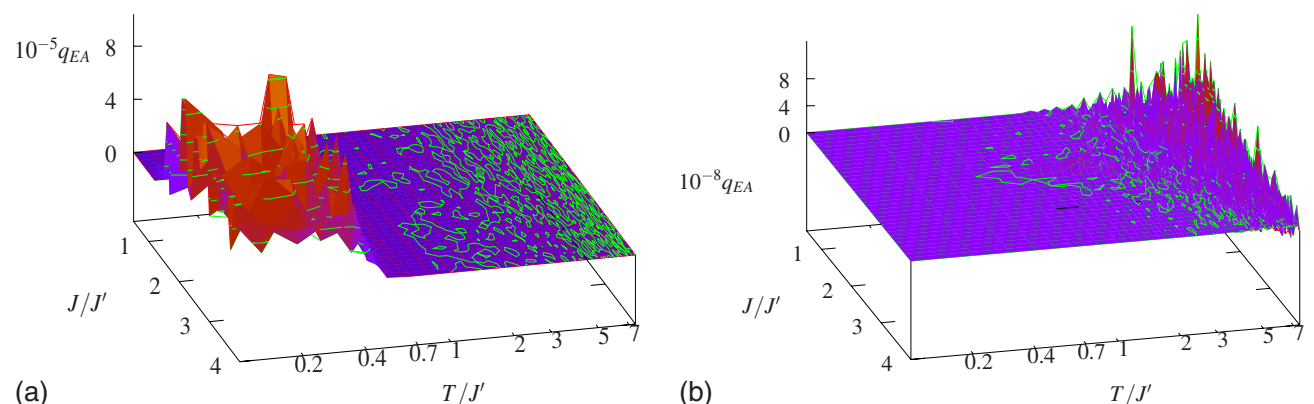

FIG. 7. (Color online) Edwards-Anderson order parameter for the $2 \mathrm{D}$ Ising model with next-nearest-neighbor interactions on a $16 \times 16$ square lattice. The figure on the left is the result of Monte Carlo simulations while the one on the right is the result of a simulation using annealing. The explosion of the Edwards-Anderson order parameter at low temperatures is an indication of the formation of a spin-glass state, which only arises in the Monte Carlo simulation without annealing.

proliferation of topological defects. The striking point is that the disordered state evolves from the ordered state when the temperature decreases. The cause of such behavior is related to a strong increase in the relaxation time for nonequilibrium domain walls when the temperature decreases. Thus, with decreasing temperature, first, the ordered state arises from a disordered state and after that at lower temperatures again a disordered state becomes more favorable. This first transition is a conventional Ising disorder-order phase transition. In the vicinity of this transition, in the ordered state, the relaxation time of the nonequilibrium domain walls is very short. Therefore, the topological defects vanish very fast. However, when temperature decreases, the height of the barriers separating the metastable minima associated with the domain walls increases. This leads to the exponential increase in the relaxation time of the nonequilibrium domain walls. In its turn, it leads to a formation of the disordered state associated with the remained domain walls, and the glassy state arises. Because of the domain-wall nature, it is convenient to describe such glassy state with the use of the proposed order parameter associated with the average length of the domain walls existing in the system. Indeed, in the present paper, we have shown that such description is consistent with the conventional one although there exists a less transparent description of the glassy state through the use of the EdwardsAnderson order parameter.

Obviously, such domain walls are always associated with metastable minima. Therefore, the zero value of entropy at low temperatures related to the state without domain walls is consistent with the exact transfer-matrix results presented in the paper. This fact indicates that the ground state at low temperatures is ordered either as stripes, $J / J^{\prime}<2$, or as the Néel antiferromagnet, $J / J^{\prime}>2$. However, the recent findings ${ }^{13,23}$ in the framework of the model under study show that the energy of topological defects and dislocations in the lattice is very close to the ground state of the system. Therefore, many locally stable (or metastable) states associated with local energy minima separated by large energy barriers may appear, forming a glasslike state.

Thus, different approaches to the analysis of the frustrated Ising model undertaken in our paper clearly and selfconsistently with each other demonstrate the possibility of the remanent quenched disorder arising at low temperatures, which manifests itself as a glassy state. Careful preparation of the lattice could result in the system being trapped in this glasslike state, which may be used as an initial state for AQC. ${ }^{13}$ The proposed order parameter based in the average domain-wall length seems to be promising in various applications in the field of AQC. Indeed, a solution to some realistic physical problem can be encoded in a certain type of Ising model with a long-range interaction between spins. The AQC-based solution of this problem could be performed via the evolution of the ground state when the interaction between spins changes. This process demands the knowledge of all spin configurations associated with the lowest-energy states of the system at any values of the model parameters. We have shown that the spin configuration can be well described by the average domain-wall length, and such an order parameter may be implemented as a useful tool for the characterization of the system evolution encountered in a AQC process. A slow variation in parameter $J / J^{\prime}$, which is, in principle, possible in the arrays of $\pi$-rings controlled by a bias voltage, can lead to an evolution of the system by transferring it adiabatically between different metastable energy minima of the low-temperature glass with nearly the same energy. Subsequent lowering and increasing of the barriers between these energy minima by changing $J / J^{\prime}$ give one a tool to move the system to a desired configuration of Ising spins that could be read out. In fact, there appears a plethora of states that is possible to use in such a way for the adiabatic quantum computations. The proposed order parameter helps to identify possible issues arising at the AQC as well as a manifold of useful configurations, when the system becomes transformed to a glassy state. Recently, a scalable design, which may be used in such a way, has been proposed and realized $^{24}$ although the range of problems that can be solved seems to be limited. One such application is a traveling salesman problem, ${ }^{25}$ which can be represented in the form of a more complicated Ising model, with a set of coupling constants. ${ }^{26}$

\section{ACKNOWLEDGMENTS}

The work was supported by the ESF network-program AQDJJ, European project CoMePhS, ISTC (Grant No. G1335), and RFBR (Project No. 08-02-00212). 
${ }^{1}$ P. Chandra and B. Doucot, Phys. Rev. B 38, 9335 (1988).

${ }^{2}$ E. Dagotto and A. Moreo, Phys. Rev. Lett. 63, 2148 (1989).

${ }^{3}$ J. Richter, Phys. Rev. B 47, 5794 (1993); J. Richter, N. B. Ivanov, and K. Retzlaff, Europhys. Lett. 25, 545 (1994).

${ }^{4}$ R. F. Bishop, D. J. J. Farnell, and J. B. Parkinson, Phys. Rev. B 58, 6394 (1998).

${ }^{5}$ L. Capriotti, F. Becca, A. Parola, and S. Sorella, Phys. Rev. Lett. 87, 097201 (2001).

${ }^{6}$ L. Siurakshina, D. Ihle, and R. Hayn, Phys. Rev. B 64, 104406 (2001).

${ }^{7}$ T. Roscilde, A. Feiguin, A. L. Chernyshev, S. Liu, and S. Haas, Phys. Rev. Lett. 93, 017203 (2004).

${ }^{8}$ J. Sirker, Z. Weihong, O. P. Sushkov, and J. Oitmaa, Phys. Rev. B 73, 184420 (2006).

${ }^{9}$ D. Schmalfuß, R. Darradi, J. Richter, J. Schulenburg, and D. Ihle, Phys. Rev. Lett. 97, 157201 (2006).

${ }^{10}$ J. R. Viana and J. R. de Sousa, Phys. Rev. B 75, 052403 (2007).

${ }^{11}$ R. Melzi, P. Carretta, A. Lascialfari, M. Mambrini, M. Troyer, P. Millet, and F. Mila, Phys. Rev. Lett. 85, 1318 (2000).

${ }^{12}$ P. Carretta, N. Papinutto, C. B. Azzoni, M. C. Mozzati, E. Pavarini, S. Gonthier, and P. Millet, Phys. Rev. B 66, 094420 (2002).

${ }^{13}$ A. O'Hare, F. V. Kusmartsev, K. I. Kugel, and M. S. Laad, Phys. Rev. B 76, 064528 (2007).

${ }^{14}$ F. V. Kusmartsev, Phys. Rev. Lett. 69, 2268 (1992).

${ }^{15}$ H. Hilgenkamp, Ariando, H.-J. H. Smilde, D. H. A. Blank, G. Rijnders, H. Rogalla, J. R. Kirtley, and C. C. Tsuei, Nature
(London) 422, 50 (2003).

${ }^{16}$ J. R. Kirtley, C. C. Tsuei, Ariando, H. J. H. Smilde, and H. Hilgenkamp, Phys. Rev. B 72, 214521 (2005).

${ }^{17}$ F. V. Kusmartsev, D. M. Forrester, and M. S. Garelli, in Physics of Superconducting Phase Shift Devices, Book of Abstracts, edited by A. Barone, E. Sarnelli, F. Tafuri, and G. Testa (University of Naples, Ischia, 2005), p. 21.

${ }^{18}$ D. M. Forrester, K. E. Kuerten, and F. V. Kusmartsev, Moldavian J. Phys. Sci. 6, 298 (2007).

${ }^{19}$ S. H. W. van der Ploeg, A. Izmalkov, Alec Maassen van den Brink, U. Hübner, M. Grajcar, E. Il'ichev, H.-G. Meyer, and A. M. Zagoskin, Phys. Rev. Lett. 98, 057004 (2007).

${ }^{20}$ S. F. Edwards and P. W. Anderson, J. Phys. F: Met. Phys. 5, 965 (1975).

${ }^{21}$ D. C. Mattis, The Theory of Magnetism II: Thermodynamics and Statistical Mechanics (Springer, New York, 1985).

${ }^{22}$ R. A. Horn and C. R. Johnson, Matrix Analysis (Cambridge University Press, Cambridge, 1990).

${ }^{23}$ A. O'Hare, F. V. Kusmartsev, M. S. Laad, and K. I. Kugel, Physica C 437-438, 230 (2006).

${ }^{24}$ V. Zakosarenko, N. Bondarenko, S. H. W. van der Ploeg, A. Izmalkov, S. Linzen, J. Kunert, M. Grajcar, E. Il'ichev, and H.-G. Meyer, Appl. Phys. Lett. 90, 022501 (2007).

${ }^{25}$ Tien D. Kieu, arXiv:quant-ph/0601151 (unpublished).

${ }^{26}$ R. Martoňák, G. E. Santoro, and E. Tosatti, Phys. Rev. E 70, 057701 (2004). 